\title{
Canadian
}

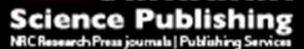

Canadian Journal of Physics

Revue canadienne de physique

\section{One-way Extraordinary Optical Transmission with Dielectric/Perforated Metal/Antiferromagnetic Structure in Terahertz Range}

\begin{tabular}{|r|l|}
\hline Journal: & Canadian Journal of Physics \\
\hline Manuscript ID & cjp-2016-0189.R1 \\
\hline Manuscript Type: & Article \\
\hline Date Submitted by the Author: & $20-$ Apr-2016 \\
\hline Complete List of Authors: & $\begin{array}{l}\text { wang, XiangGuang; Laboratory for Photonic and Electronic Bandgap } \\
\text { Materials, Ministry of Education, School of Physics and Electronic } \\
\text { Engineering } \\
\text { Zhang, Qiang; Laboratory for Photonic and Electronic Bandgap Materials, } \\
\text { Ministry of Education, School of Physics and Electronic Engineering, Harbin } \\
\text { Normal University } \\
\text { Fu, ShuFang; Laboratory for Photonic and Electronic Bandgap Materials, } \\
\text { Ministry of Education, School of Physics and Electronic Engineering } \\
\text { Song, Yuling; Laboratory for Photonic and Electronic Bandgap Materials, } \\
\text { Ministry of Education, School of Physics and Electronic Engineering } \\
\text { Wang, Xuanzhang; Laboratory for Photonic and Electronic Bandgap } \\
\text { Materials, Ministry of Education, School of Physics and Electronic } \\
\text { Engineering } \\
\text { Zhou, Sheng; Key Laboratory for Photonic and Electronic Bandgap } \\
\text { Materials, Ministry of Education, School of Physics and Electronic } \\
\text { Engineering, ; Department of Electrical and Computer Engineering }\end{array}$ \\
\hline Keyword: & $\begin{array}{l}\text { One-way, Antiferromagnetic, Perforated metal, Terahertz, Surface plasmon } \\
\text { polaritons }\end{array}$ \\
\hline
\end{tabular}




\section{One-way Extraordinary Optical Transmission with}

\section{Dielectric/Perforated Metal/Antiferromagnetic Structure in}

\section{Terahertz Range*}

Xiang guang Wang ${ }^{1}$, Qiang zhang ${ }^{1,2}$, Shufang $\mathrm{Fu}^{1}$, Yuling Song ${ }^{1}$, xuan zhang Wang ${ }^{1}$ and Sheng Zhou ${ }^{1,3,+}$

${ }^{1}$ Key Laboratory for Photonic and Electronic Bandgap Materials, Ministry of Education, School of Physics and Electronic Engineering, Harbin Normal University, Harbin 150025, China

${ }^{2}$ Harbin University of Science and Technology, Harbin 150025, China

${ }^{3}$ University of California, Riverside, Riverside, 92521, United states of America

\section{Abstract}

The one-way optical effect in a dielectric/perforated metal/antiferromagnetic (DPMA) sandwich structure in the terahertz range is studied. Theoretical modeling, which is based on the effective medium theory and the transmission matrix method, is conducted. The simulation results show the one-way effect occurs within a wide frequency range and for various incident angles with a fixed external magnetic field. In addition, this behavior is examined for various DPMA structural characteristics, such as different dielectric materials, film thicknesses, and perforated-metal hole sizes and spacings. The developed sandwich structures can aid the design of subminiature optical isolators for application in terahertz range.

PACS: 42.25.Bs, 75.50.Ee, 07.57.-c, 73.20.Mf

Keywords: One-way, Antiferromagnetic, Perforated metal, Terahertz(THz), Surface Plasmon Polaritons (SPP)

\section{Introduction}

In recent years, radiation in the terahertz $(\mathrm{THz})(0.1-10 \mathrm{THz})$ range has attracted considerable interest, because of its broad range of applications in science and

\footnotetext{
* Project supported by the National Natural Science Foundation of China (Grant No.11204056, 11104050, 11304068), the Science and Technology Research Project of department of education of Heilongjiang Province, China (Grant No. 12521154) and Graduate Students' Research Innovation Project of Harbin Normal University (HSDSSCX2015-21).

+Corresponding author: zhousheng_wl@,163.com
} 
technology [1], e.g., in high-speed communication [2], nondestructive spectroscopy [3], sensing [4], and imaging [5, 6]. Further, the resonant frequencies of typical antiferromagnetic materials (AF) are distributed over the $\mathrm{THz}$ range. With the development of laser technology and materials science, the optical properties of AF materials are receiving increasing attention as a result of their unique nonreciprocity, and also because of the one-way [7], Kerr, and Faraday effects [8, 9]. In early studies, the frequencies of $\mathrm{AF}$ (such as $\mathrm{Bi}: \mathrm{YIG}[10]$ and $\mathrm{MnO}$ [11]) were thought to be located in the visible and near-infrared ranges. However, recent research has shown that these frequencies are near the THz range. Further, Kampfrath $[12,13]$ has found that the spin-wave and electromagnetic coupling in $\mathrm{NiO}$ film can produce $\mathrm{THz}$ radiation. This discovery has provided experimental evidence for the capacity of AF materials for $\mathrm{THz}$ radiation generation. Notably, light localization can be realized through a multilayered medium [14]; however, this is not conducive to device miniaturization and integration. Therefore, a structure combining AF within a small space is required in order to enhance the AF linear effect [7-9] and to offer advantages as regards device miniaturization.

Pure metallic surfaces can no longer confine the subwavelength scale in the $\mathrm{THz}$ range, because of the weak coupling between the electromagnetic field and the electrons in metal [15]. Since Ebbesen's group [16] first experimentally observed the extraordinary light (EOT) phenomenon in perforated metal (PM) film, PM film has been used to realize the optical properties of general dielectric multilayer film [17]. In such films, the $\mathrm{THz}$ waves can be supported and confined by structured metallic surfaces known as spoof surface plasmon polaritons (SPP) [18].

Khanikaev [7] found that the one-way propagation of light can be realized by placing ferromagnetic films on both sides of the square hole arrays of a subwavelength metal film, and reported an example of combined PM and magnetic materials. In addition, theoretical and experimental research has shown that PM materials such as gold or silver [18] are typically good conductors in the $\mathrm{THz}$ frequency region. The optical properties of PM thin films are primarily based on the excitation of SPP on a metal surface, as well as coupling-localized SPP with an electric dipole [19]. The electromagnetic field is enhanced through combination with 
a waveguide action, which greatly reinforces the light diffraction. The stimulated SPP can be regulated by changing the arrangement rules of the subwavelength hole arrays.

The PM-to-AF structure is introduced due to simplify the structure of the photonic crystal. A multilayer structure composed of an AF and a PM layer, where the AF film thickness ranges from tens to hundreds of micrometers, has unique properties. This means that it is relatively easy to generate the required phenomena in experiment and in practical application. Further, the influence of quantum effects can be ignored, because the PM and AF layer sizes are of the order of microns, which is beyond the theoretical level at which quantum effects would influence the optical properties of the PM/AF multilayer. The optical properties of the AF materials can be tuned by varying an external magnetic field by approximately $0.1 \mathrm{~T}[8,9]$. The plasma frequency of the metal material is approximately $10^{16} \mathrm{~Hz}$ and the corresponding electron cyclotron frequency is $10^{11} \mathrm{~Hz} / \mathrm{T}$ which is significantly smaller than the plasma frequency of the metal. The optical properties of the metal would be affected by a magnetic field intensity of approximately $10^{4} \mathrm{~T}$, but such a high magnetic field cannot be achieved under the present experimental conditions. Therefore, the external static magnetic field affects the optical properties of the AF materials only and does not affect those of the PM.

Here we focus on the linear optical properties in a dielectric/PM/AF (DPMA) sandwich structure, and in particular on investigating the correction for the magnetopolaron and the electromagnetic field on the PM surface. By regulating the sub-wavelength holes to affect the interaction between the AF magnetic dipole and the PM SPP, the properties of the electromagnetic waves, such as the abnormal optical transmission and the one-way mode, are found to increase. The one-way behavior indicates that electromagnetic waves can be unidirectional within certain frequency ranges. That is, they are permitted to propagate in one direction only, either forward or backward, while propagation in the opposite direction is prohibited or significantly suppressed. By controlling the magnetic field, incident light is located in the AF layer. In this way, we can enhance the nonreciprocal of the AF system linear effect. Optical isolator (ISO) [20] functionality is realized by the nonreciprocity of the PM and AF, because of the abnormal light transmission properties of PM [18]. Hence, we can 
design an ISO that is smaller than traditional miniaturized ISOs. Intensive study of the ISO performance indicates that the insertion loss parameter range is approximately $0.14-0.5 \mathrm{~dB}$, and the reverse isolation is approximately $30-45.8 \mathrm{~dB}$.

The paper is structured as follows. The section 2 shows the theoretical modeling where include the linear transmission matrix method and the effective medium theory. The section 3 presents the theoretical results and implications graphically. Finally, conclusions are shown in section 4.

\section{THEORETICAL FORMALISM}

Typical sandwich layered structures with different symmetries are constructed as shown in Fig. 1. In this study, a novel DPMA sandwich structure is designed in order to realize an optical isolator (ISO). We use a Cartesian coordinate system with the $y$ axis normal to the surface of the sandwich structure. The external magnetic field $\vec{H}$ and the AF anisotropic field are parallel along the $z$ axis. The $x-y$ plane is the incidence plane, and $I, R$, and $T$ are the incident, reflected, and transmitted waves, respectively, while $\theta$ represents the angle of incidence. The thicknesses of the PM, $\mathrm{AF}$, and dielectric layers are represented by $d_{m s}, d_{a}$, and $d_{1}$, respectively, and their dielectric constants are $\varepsilon_{m s}, \varepsilon_{a}$, and $\varepsilon_{1}$, respectively. The magnetic permeability of the nonmagnetic media is taken as $\mu_{0}=1$. The PM layer is a perfect conductor layer with an array of holes. We assume a square cross section of $a \times a$ for the holes and a square array of side $d$. The holes and their spacing are significantly smaller than the radiation wavelength, that is, $a<d<<\lambda_{0}$. An incident wave excites several waveguide modes in the holes, but the fundamental mode is dominant because it exhibits the weakest decay. Both the electric $\vec{E}$ and $\vec{H}$ fields are zero inside the conductor, but in the holes $\vec{E}$ has the form

$$
\vec{E}=E_{0}[0,1,0] \sin (\pi x / a) \exp \left(i k_{y}^{m s} y-i \omega t\right),
$$

where $0<x<a, 0<y<a$, and $E_{0}$ is a constant. Further, $i=\sqrt{-1}, \omega$ is the frequency, $t$ is time, and $k_{y}^{m s}=i \sqrt{\pi^{2} / a^{2}-\varepsilon_{h} \mu_{h} k_{0}^{2}}$, where $k_{0}$ is the free-space wave vector and $\varepsilon_{h}$ and $\mu_{h}$ are the permittivity and permeability of any material that may be filling the holes. We take the $y$ axis to be the surface normal. The electromagnetic wave $H$ fields along the $x$ and $z$ axes are defined as $H_{x}$ and $H_{z}$, respectively. From the linear algebra matrix changes and the theory of Pendry et al. 
[18], we deduce

$$
\begin{gathered}
\mu_{m s}=\left(\begin{array}{ccc}
\mu_{x x}^{m s} & 0 & 0 \\
0 & \mu_{y y}^{m s} & 0 \\
0 & 0 & \mu_{z z}^{m s}
\end{array}\right), \\
\varepsilon_{m s}=\left(\begin{array}{ccc}
\varepsilon_{x x}^{m s} & 0 & 0 \\
0 & \varepsilon_{y y}^{m s} & 0 \\
0 & 0 & \varepsilon_{z z}^{m s}
\end{array}\right),
\end{gathered}
$$

where $\mu_{y}=\infty, \mu_{x}=\mu_{z}=\frac{8 a^{2} \mu_{h}}{\pi^{2} L^{2}}, \varepsilon_{y}=\infty$, and $\varepsilon_{x}=\varepsilon_{z}=\frac{\pi^{2} L^{2} \varepsilon_{h}}{8 a^{2}}\left(1-\frac{\pi^{2} c_{0}^{2}}{a^{2} \omega^{2} \varepsilon_{h} \mu_{h}}\right)$.

The AF linear magnetic permeability is $[21,22]$

$$
\begin{gathered}
\vec{\mu}=1+\vec{\chi}=\left(\begin{array}{ccc}
1+\chi_{x x}^{(1)} & \chi_{x y}^{(1)} & 0 \\
-\chi_{y x}^{(1)} & 1+\chi_{y y}^{(1)} & 0 \\
0 & 0 & 1
\end{array}\right)=\left(\begin{array}{ccc}
\mu_{1} & i \mu_{2} & 0 \\
-i \mu_{2} & \mu_{1} & 0 \\
0 & 0 & 1
\end{array}\right), \\
\mu_{1}=1+\omega_{m} \omega_{a}\left[\frac{1}{\omega_{r}^{2}-\left(\omega-\omega_{0}\right)^{2}}+\frac{1}{\omega_{r}^{2}-\left(\omega+\omega_{0}\right)^{2}}\right], \\
\mu_{2}=\omega_{m} \omega_{a}\left[\frac{1}{\omega_{r}^{2}-\left(\omega-\omega_{0}\right)^{2}}-\frac{1}{\omega_{r}^{2}-\left(\omega+\omega_{0}\right)^{2}}\right],
\end{gathered}
$$

where $\omega_{0}=\gamma H_{0}, \omega_{m}=\gamma M_{0}, \omega_{a}=\gamma H_{a}+i \tau \omega, \omega_{e}=\gamma H_{e}, \omega_{r}=\left[\omega_{a}\left(2 \omega_{e}+\omega_{a}\right)\right]^{1 / 2}$. The external magnetic field is $H_{0}$. And other the physical parameters are also well known, of which the exchange field is $H_{e}$, the anisotropy field is $H_{a}$, the sublattice magnetization is $M_{0}$, the gyromagnetic ratio is $\gamma$, the damping coefficient is $\tau$.

The transfer-matrix method has been widely applied to sandwich structures. Any incident plane electromagnetic wave can be decomposed into a transverse magnetic (TM) wave, with $H$ normal to the incident plane, and a transverse electric (TE) wave [23], with $E$ normal to the incident plane. However, the TM wave does not couple with the magnetization in the AF. Therefore, we suppose that the incident wave is the TE wave. The wave solution in various spaces can be determined from 


$$
E_{z}=\exp \left(i k_{x} x-i \omega t\right) \times\left\{\begin{array}{c}
I_{0} \exp \left(i k_{0 y} y\right)+R_{0} \exp \left(-i k_{0 y} y\right), \\
(\text { in the upper space) } \\
A_{j} \exp \left(i k_{j y} y\right)+B_{j} \exp \left(-i k_{j y} y\right), \\
\text { (in the } j \text { layer) } \\
T_{0} \exp \left(i k_{y} y\right), \\
\text { (in the lower space) }
\end{array}\right.
$$

Where $j=1, m s$, or $a$, corresponding to the dielectric, PM, and AF layers, respectively. The wave-numbers are $k_{0}=\left[(\omega / c)^{2}-k_{x}^{2}\right]^{1 / 2}, k_{1}=\left[\varepsilon_{1}(\omega / c)^{2}-k_{x}^{2}\right]^{1 / 2}$, $k_{a}=\left[\varepsilon_{a} \mu_{v}(\omega / c)^{2}-k_{x}^{2}\right]^{1 / 2}$, and $k_{m s}=i\left[\pi^{2} / a^{2}-\varepsilon_{h} \mu_{h} k_{0 m s}^{2}\right]^{1 / 2}$, with $k_{x}=(\omega / c) \sin \theta$. The AF effective permeability is $\mu_{v}=\left(\mu_{x x}^{2}+\mu_{x y}^{2}\right) / \mu_{x x} . I_{0}, R_{0}$, and $T_{0}$ indicate the incident, reflected, and transmitted wave amplitudes, respectively. The wave magnetic field can be derived from $\nabla \times \vec{E}=i \omega \mu_{0} \vec{H}$ ( $\mu_{0}$ is the vacuum magnetic permeability) in nonmagnetic media and $\nabla \times \vec{E}=i \omega \mu_{0} \vec{\mu} \cdot \vec{H}$ in the AF. Because continuous boundary conditions of $E_{z}$ and $H_{x}$ at interfaces are assumed, the $x$ component of $\vec{H}$ can be expressed in the various media as

$$
H_{x}=\frac{\mathrm{e}^{i\left(k_{x} x-\omega t\right)}}{\omega}\left\{\begin{array}{rr}
k_{0 y}\left[I_{0} \exp \left(i k_{0 y} y\right)-R \exp \left(-i k_{0 y} y\right)\right], \\
\quad \text { (in the upper space) } \\
k_{j y}\left[A_{j} \exp \left(i k_{j y} y\right)-B_{j} \exp \left(-i k_{j y} y\right)\right], \\
\text { (in the dielectric layer) } \\
\frac{1}{\mu_{x x}}\left[k_{m s y} A_{m s} \exp \left(i k_{m s y} y\right)-k_{m s y} B_{m s} \exp \left(-i k_{m s y} y\right)\right], \\
\text { (in the PM layer) } \\
\left.\frac{k_{a y}}{\mu_{v}}\left(1+\delta_{a}\right) A_{a} \exp \left(i k_{j y} y\right)-\left(1-\delta_{a}\right) B_{a} \exp \left(-i k_{a y} y\right)\right], \\
k_{y} T_{0} \exp \left(i k_{y} y\right), & \text { (in the AF layer) }
\end{array}\right.
$$

Where $\delta_{a}=i \mu_{x y} k_{x} / \mu_{y y} k_{a y}$. Although we do not apply the $y$ component of $\vec{H}$, under the boundary conditions, this component in the DPMA is used to determine the AF, with

$$
\begin{aligned}
& H_{y}=\frac{k_{x}}{\omega \mu_{v}}\left\{\left(\delta_{a}^{\prime}-1\right) A_{a} \exp \left(i k_{a y} y\right)\right. \\
& \left.\left.-\left(\delta_{a}^{\prime}+1\right) B_{a} \exp \left(-i k_{a y} y\right)\right]\right\} \exp \left(i k_{x} x-i \omega t\right),
\end{aligned}
$$


where $\delta_{a}^{\prime}=\mu_{x y} k_{a y} / \mu_{x x} k_{x}$. From the boundary conditions, we can find the relation between the wave amplitudes in any pair of adjacent media. The amplitude relation at the surface is

$$
\left(\begin{array}{c}
I_{0} \\
R
\end{array}\right)=T_{t 1}\left(\begin{array}{c}
A_{1} \\
B_{1}
\end{array}\right)
$$

where $T_{t 1}=\frac{1}{2 k_{t y}}\left(\begin{array}{ll}k_{t y}+k_{1 y} & k_{t y}-k_{1 y} \\ k_{t y}-k_{1 y} & k_{t y}+k_{1 y}\end{array}\right)$. The amplitudes in the dielectric layer above and adjacent to the AF and those in the AF are conjoined by

$$
\left(\begin{array}{c}
A_{1} \\
B_{1}
\end{array}\right)=T_{1 m s}\left(\begin{array}{l}
A_{m s} \\
B_{m s}
\end{array}\right)
$$

where

$$
T_{1 m}=\frac{1}{2 \mu_{x x} k_{1 y}} \times\left(\begin{array}{cc}
\left(\mu_{x x} k_{1 y}+k_{m y}\right) \exp \left(-i k_{1 y} d_{1}\right) & \left(\mu_{x x} k_{1 y}-k_{m y}\right) \exp \left(-i k_{1 y} d_{1}\right) \\
\left(\mu_{x x} k_{1 y}-k_{m y}\right) \exp \left(i k_{1 y} d_{1}\right) & \left(\mu_{x x} k_{1 y}+k_{m y}\right) \exp \left(i k_{1 y} d_{1}\right)
\end{array}\right) .
$$

The amplitudes in the AF and those in the layer below and adjacent to the AF are conjoined by

$$
\left(\begin{array}{c}
A_{m s} \\
B_{m s}
\end{array}\right)=T_{m s a}\left(\begin{array}{l}
A_{a} \\
B_{a}
\end{array}\right)
$$

where

$$
T_{m s a}=\frac{1}{2} \times\left(\begin{array}{cc}
\left(1+\mu_{x x} \delta_{m s}^{+}\right) \exp \left(-i k_{m s y} d_{m s}\right) & \left(1-\mu_{x x} \delta_{m s}^{-}\right) \exp \left(-i k_{m s y} d_{m s}\right) \\
\left(1-\mu_{x x} \delta_{m s}^{+}\right) \exp \left(i k_{m s y} d_{m s}\right) & \left(1+\mu_{x x} \delta_{m s}^{-}\right) \exp \left(i k_{m s y} d_{m s}\right)
\end{array}\right)
$$

with $\delta_{m s}^{ \pm}=\frac{k_{a y}}{\mu_{v} k_{m s y}}\left(1 \pm \delta_{a}\right)$. Finally, we find the relation at the bottom surface to be

$$
\left(\begin{array}{c}
A_{a} \\
B_{a}
\end{array}\right)=T_{a b}\left(\begin{array}{c}
T_{0} \\
0
\end{array}\right)
$$

Where

$$
T_{a b}=\frac{1}{2} \times\left(\begin{array}{cc}
\left(1-\delta_{a}+\frac{\mu_{v} k_{b y}}{k_{a y}}\right) \exp \left(-i k_{a y} d_{a}\right) & 0 \\
0 & \left(1+\delta_{a}-\frac{\mu_{v} k_{b y}}{k_{a y}}\right) \exp \left(i k_{a y} d_{a}\right)
\end{array}\right)
$$

According to the above equations, the relation between the reflection, transmission, and incident amplitudes is 


$$
\left(\begin{array}{c}
I_{0} \\
R
\end{array}\right)=T_{t 1} T_{1 m s} T_{m s a} T_{a b}\left(\begin{array}{c}
T_{0} \\
0
\end{array}\right)=\prod\left(\begin{array}{c}
T_{0} \\
0
\end{array}\right)
$$

When the light travels in the positive direction through the DPMA structure, the transmission rate is $\Pi$. By studying the one-way behavior of the sandwich structure, we obtain the Transfer Matrix Method (TMM) of the AF/PM/dielectric structure. According to the amplitude relation at the surface, the matrix is

$$
\left(\begin{array}{l}
I_{0} \\
R
\end{array}\right)=\frac{1}{2}\left(\begin{array}{cc}
1+\frac{k_{a y}\left(1+\delta_{a}\right)}{k_{t y} \mu_{v}} & 1-\frac{k_{a y}\left(1-\delta_{a}\right)}{k_{t y} \mu_{v}} \\
1-\frac{k_{a y}\left(1+\delta_{a}\right)}{k_{t y} \mu_{v}} & 1+\frac{k_{a y}\left(1-\delta_{a}\right)}{k_{t y} \mu_{v}}
\end{array}\right)\left(\begin{array}{l}
A_{a} \\
B_{a}
\end{array}\right)=T_{t a}\left(\begin{array}{l}
A_{a} \\
B_{a}
\end{array}\right),
$$

where $\delta_{a}=\frac{\mu_{a x y} k_{x}}{\mu_{a y y} k_{a y}}$.The amplitudes in the PM layer above and adjacent to the AF and those in the $\mathrm{AF}$ are conjoined by

$$
\left(\begin{array}{l}
A_{a} \\
B_{a}
\end{array}\right)=\frac{\mu_{v} k_{m s y}}{2 \mu_{x x} k_{a y}}\left(\begin{array}{cc}
\left(\mu_{x x} \delta_{m s}^{-}+1\right) \mathrm{e}^{-i k_{a y} d_{a}} & \left(\mu_{x x} \delta_{m s}^{-}-1\right) \mathrm{e}^{-i k_{a y} d_{a}} \\
\left(\mu_{x x} \delta_{m s}^{+}-1\right) \mathrm{e}^{i k_{a y} d_{a}} & \left(\mu_{x x} \delta_{m s}^{+}+1\right) \mathrm{e}^{i k_{a y} d_{a}}
\end{array}\right)\left(\begin{array}{l}
A_{m s} \\
B_{m s}
\end{array}\right)=T_{a m s}\left(\begin{array}{l}
A_{m s} \\
B_{m s}
\end{array}\right) .
$$

The amplitudes in the AF and those in the layer below and adjacent to the dielectric are conjoined by

$$
\left(\begin{array}{l}
A_{m s} \\
B_{m s}
\end{array}\right)=\frac{1}{2 k_{m y}}\left(\begin{array}{cc}
\left(k_{m s y}+\mu_{x x} k_{1 y}\right) \mathrm{e}^{-i k_{m y} d_{m s}} & \left(k_{m s y}-\mu_{x x} k_{1 y}\right) \mathrm{e}^{-i k_{m y} d_{m s}} \\
\left(k_{m s y}-\mu_{x x} k_{1 y}\right) \mathrm{e}^{i k_{m y} d_{m s}} & \left(k_{m s y}+\mu_{x x} k_{1 y}\right) \mathrm{e}^{i k_{m y} d_{m s}}
\end{array}\right)\left(\begin{array}{l}
A_{1} \\
B_{1}
\end{array}\right)=T_{m s 1}\left(\begin{array}{l}
A_{1} \\
B_{1}
\end{array}\right) .
$$

Finally, we find $\delta_{a}=\frac{\mu_{x y} k_{x}}{\mu_{y y} k_{a y}}$ and the relation at the bottom surface to be

$$
\left(\begin{array}{l}
A_{1} \\
B_{1}
\end{array}\right)=\frac{1}{2}\left(\begin{array}{cc}
\left(1+\frac{k_{b y}}{k_{1 y}}\right) \exp \left(-i k_{1 y} d_{1}\right) & 0 \\
0 & \left(1-\frac{k_{b y}}{k_{1 y}}\right) \exp \left(i k_{1 y} d_{1}\right)
\end{array}\right)\left(\begin{array}{c}
T_{0} \\
0
\end{array}\right)=T_{1 b}\left(\begin{array}{c}
T_{0} \\
0
\end{array}\right)
$$

Thus we obtain the matrix equation comprising the reflection, transmission, and incident amplitudes

$$
\left(\begin{array}{c}
I_{0}^{\prime} \\
R^{\prime}
\end{array}\right)=T_{t a} T_{a m s} T_{m s 1} T_{1 b}\left(\begin{array}{c}
T_{0} \\
0
\end{array}\right)=\Pi^{\prime}\left(\begin{array}{c}
T_{0} \\
0
\end{array}\right)
$$

When the light travels in a positive direction through the AF/PM/dielectric structure (i.e., in a negative direction through the DPMA structure), the transmission rate is $\Pi^{\prime}$. 
It is well known that EOT in PM is inherently related to excitation of SSPs [18, 24], however, one-way parameters do not satisfy ISO standards. In this paper, the required results are achieved using the $\mathrm{AF} / \mathrm{PM} /$ dielectric structure, in which the one-way parameter is

$$
\Gamma=\Pi-\Pi^{\prime}
$$

\section{NUMERICAL RESULTS AND DISCUSSION}

An $\mathrm{AF} / \mathrm{PM} /$ dielectric sandwich structure is designed where the dielectric layer is $\mathrm{ZrF}_{2}$, having $\varepsilon_{1}=27$. We use $M n F_{2}$ as the AF material in the designed structures. The physical parameters are well known $[25,26]$, of which the exchange field is $H_{e}=550 k G$, the anisotropy field is $H_{a}=7.87 k G$, the sublattice magnetization is $M_{0}=7.87 \mathrm{kG}$, the gyromagnetic ratio is $\gamma=1.97 \times 10^{10} \mathrm{rad} \cdot \mathrm{s}^{-1} \cdot k G^{-1}$, the damping coefficient is $\tau=0.001$, and $\varepsilon_{a}=5.5$. The resonant frequency is $\omega / 2 \pi c=9.7588 \mathrm{~cm}^{-1}$ for a zero external magnetic field. The layer thicknesses are fixed as $d_{1}=89.9 \mu \mathrm{m}, d_{m s}=4.3 \mu \mathrm{m}$, and $d_{a}=109.2 \mu \mathrm{m}$, excluding the case in which the layer-thickness dependence is examined. The PM hole size and spacing are $a=101.3 \mu m$ and $L=304.1 \mu m$, respectively.

We first discuss the transmission in an $\mathrm{MnF}_{2} / \mathrm{Ag} / \mathrm{ZrF}_{2}$ sandwich structure. The linear transmission spectra are shown in Fig. 2. The incident light is oriented in different directions and, thus, we illustrate the transmission of a single AF film against varying $\omega$ and $\theta$ in Fig. 2(a) and Fig. 2(b) (positive and negative transmission rates, respectively). The $t$ symbol indicates positive incidence transmission through the $\mathrm{AF} / \mathrm{PM} /$ dielectric structure, while $t^{\prime}$ corresponds to negative incidence. For the same structure, the positive and negative transmission peaks have different frequency ranges, because they lie on the incident wave vector of the upper PM layer (Eq. 1). Further, $k_{0}$ in Eq. 1 is the incident wave vector of the PM surface, so the positive and negative directions of the incident wave vector of the PM layer are affected by the dielectric and AF layers. It is evident that their respective maxima are $t=0.985166$ and $t^{\prime}=0.994610$. Using the acquired data, we can obtain the difference between the positive and negative transmissions as shown in Fig. 2(c). The frequencies at which one-way behavior is most obvious are $\omega / 2 \pi \mathrm{c}=14.5 \mathrm{~cm}^{-1}$ and 
$\omega / 2 \pi \mathrm{c}=19.7 \mathrm{~cm}^{-1}$. The calculation results confirm that one-way transmittance occurs within a very large $\theta$ range. Thus, we can obtain one-way behavior for arbitrary $\theta$.

Secondly, in order to study the influence of the PM $a$ and $L$ on the unipolarity of the DPMA structure, $d_{m s}$ was set to $4.3 \mu \mathrm{m}$. The influence of $a$ on the unidirectional spectrum is shown in Fig. 3(a). It is apparent that, with increasing $a$, the unidirectional parameters first increase and then decrease. When $a=101.3 \mu \mathrm{m}$, the one-way behavior reaches a peak. Fig. 3(b) reveals the influence of $L$ on the unidirectional spectrum. As $L$ is increased, the unidirectional coefficient first increases and then decreases. When $L=304.1 \mu \mathrm{m}$ is obtained, the unidirectional coefficient reaches a peak. The reason for this is that the PM $a$ and $L$ affect the metal's relative permittivity $\varepsilon^{m s}$ and magnetic permeability $\mu^{m s}$ (which are calculated using Eqs. 2 and 3), and play an important role in determining the light propagation in the structure.

Next, we discuss the one-way effect for the dielectric layer structure. A variety of dielectric layers with different $\theta$ and $\omega$ were examined. In Fig. 4, we show the observed one-way effect for the dielectric $\mathrm{ZrO}_{2} \quad(\varepsilon=8.0)$ and $\mathrm{ZrF}_{2} \quad(\varepsilon=27.0)$ materials. In Fig. 4(a), the unidirectional peak changes with $\theta$ for the $\mathrm{ZrO}_{2}$ dielectric layer. However, in the case of $\mathrm{ZrF}_{2}$, the unidirectional peak does not change with $\theta$. As shown in Fig. 4(b), the unidirectional peak moves to smaller $\omega$ for increased $\varepsilon$, when $\theta$ is $20^{\circ}$ and $80^{\circ}$. Thus, depending on our requirements, we can select the appropriate dielectric material.

We also consider the unidirectional behavior as a function of the film thickness of an $\mathrm{MnF}_{2} / \mathrm{Ag} / \mathrm{ZrF}_{2}$ structure, as shown in Fig. 5. In Fig. 5(a), the one-way parameters vary periodically with increasing $d_{1}$, while the peak decreases periodically. For $d_{1}=89.9 \mu \mathrm{m}$, the one-way parameters yield the highest unipolarity. Note that $d_{m s}$ is smaller than $d_{1}$, and the unidirectional results for varying $d_{m s}$ are shown in Fig. 5(b). When $d_{m s}=4.3 \mu m$, the one-way parameters yield a peak. Further, the unidirectional peak decreases with increased film thickness. Finally, the one-way parameters vary cyclically with changes in $d_{a}$. When $d_{a}=109.2 \mu \mathrm{m}$, the one-way parameters are at the highest point, as shown in Fig. 5(c). The one-way behavior varies cyclically because it reflects the relationship between the film thickness and the incident wavelength $\lambda$. 
Finally, we discuss the influence of the external field $H_{0}$ on the positive and negative unipolarity. Changing $H_{0}$ can affect the AF resonance interval, and also affects the unipolarity of the structure, as shown in Fig. 6. Only through variation of $H_{0}$, a rotation is observed, that the unipolarity suddenly changes from positive to opposite at the same frequency marked by a black line in the figure.

\section{CONCLUSION}

We have introduced a new sandwich structure material comprised of dielectric/perforated metal/antiferromagnetic layers effective in the terahertz wavelength range. By theoretically combining the perforated metal with an antiferromagnetic material and exploring the one-way optical transmission using the transfer matrix and boundary condition method, we have realized unipolarity at different frequency ranges. Also, by adjusting the size and spacing of the perforated metal holes, this material can be tuned to exhibit the optimum unipolarity parameters. The unipolarity peak does not change with increased incident angle for a $\mathrm{ZrF}_{2}$ dielectric layer. However, this peak does vary with increased incident angle in the case of a $\mathrm{ZrO}_{2}$ dielectric layer. Because of the relationship between the structure thickness and the incident frequency, the change in unipolarity with varying thickness is periodic. By altering the external magnetic field, we have determined that the unipolarity and the mono-direction are reversely oriented at the same frequency. Thus, we can obtain a structure with the optimal unidirectional effect by accommodating an additional static magnetic field and setting the incident angle, the dielectric material, and the size and spacing of the perforated metal holes.

In this study, we provide a strong theoretical basis for the development of an optical isolator. This will address the problem of reverse energy drawbacks in contemporary remote fiber-optic transmission, promoting the development of advanced modern optical communication systems. 


\section{ACKNOWLEDGMENTS}

Project supported by the National Natural Science Foundation of China (Grant No.11204056,11104050,11304068), the Science and Technology Research Project of department of education of Heilongjiang Province, China (Grant No. 12521154) and Graduate Students' Research Innovation Project of Harbin Normal University (HSDSSCX2015-21). 


\section{REFERENCES}

[1] M. Sherwin, C. Schmuttenmaer, and P. Bucksbaum. Opportunities in THz Science: Report of a DOE-NSF-NIH Workshop; Arlington, VA, 2004 12-14.. doi:10.2172/ 899222.

[2] M. Lee and M. C. Wanke. Science. 2007 316(5821), 64. doi:10.1126/science. 1141012.

[3] R. A. Stead, A. K. Mills, and D. J. Jones. J. Opt. Soc. Am. B. 2012 29(10), 2861. doi:10.1364/JOSAB.29.002861.

[4] J. F. O'Hara, W. Withayachumnankul, and I. Al-Naib, J. Infrared Millimeter Terahertz Waves. 2012 33(3), 245.doi:10.1007/s10762-012-9878-x.

[5] T. Ouchi, K. Kajiki, T. Koizumi, T. Itsuji, Y. Koyama, R. Sekiguchi, O. Kubota, and K. Kawase. J. Infrared Millim. THz W. 2014 35(1), 118.doi:10.1007/ s1072-013-0004-5.

[6] H. Nakanishi, S. Fujiwara, K. Takayama, I. Kawayama, H. Murakami, and M. Tonouchi. Appl. Phys. Express. 2012 5(11), 112301. doi:10.1143/APEX.5. 112301.

[7] Alexander B. Khanikaev, S. Hossein Mousavi, Gennady Shvets, and Yuri S. Kivshar. Phys. Rev. Lett . 2010 105(12-17), 126804.doi:10.1103/PhysRevLett. 105.126804.

[8] X. Z. Wang. J. Phys.: Condens. Matters. 2005 17(36), 5447. doi:10.1088/ 0953-8984/17/36/001

[9] Xuan-Zhang Wang and Yan Zhao. J. Appl. Phys. 2013 113(2), 023501. doi:10.1063/1.4773476.

[10]B. Heinrich, C. Burrowes, E. Montoya. Phys. Rev. Lett. 2011 107(6), 066604. doi:10.1103/PhysRevLett.107.066604.

[11]T. Moriyasu ,S. Wakabayashi and T. Kohmoto. J. Infrared Millim. THz W. 2013 34(3), 277.doi:10.1007/s10762-013-9963-9.

[12] Takuya Satoh, Sung-Jin Cho, Tsutomu Shimura, Kazuo Kuroda, Hiroaki Ueda, Yutaka Ueda, and Manfred Fiebig. J. Opt. Soc. Am. B. 2010 27(7), 1421. doi:10.1364/JOSAB.27.001421.

[13]Tobias Kampfrath, Alexander Sell, Gregor Klatt, Alexej Pashkin, Sebastian Mahrlein,Thomas Dekorsy, Martin Wolf, Manfred Fiebig, Alfred Leitenstorfer and Rupert Huber. Nature Photonics. 2011 5(1), 31.doi:10.1038/nphoton.2010. 259.

[14]Aroua Walid ,AbdelMalek Fathi, Haxha Shyqyri. IEEE J Quantum Elect. 2014 50(8), 633. doi: 10.1109/JQE.2014.2330762.

[15]Q. Gan and F. J. Bartoli. IEEE J. Sel. Top. Quantum Electron. 2011 17(1),102 . doi:10.1109/JSTQE.2010.2044635.

[16]T. W. Ebbesen, H. J. Lezec, H. F. Ghaemi, T. Thio , and P. A. Wolff. Nature. 1998 391(6668), 667. doi:10.1038/35570.

[17]Konstantin Y. Bliokh, Yury P. Bliokh, Valentin Freilikher, Sergey Savel ,and Franco Nori. Rev. Mod. Phys. 2008 80(4), 1201.doi:10.1103/RevModPhys. 80.1201 . 
[18]J. B. Pendry, L. Martín-Moreno, and F. J. Garcia-Vidal. Science. 2004 305(5685) 847.doi: 10.1126/science.1098999.

[19] Shan Wu, Lin Zhou, Qianjin Wang, Xuejin Zhang, and Yong yuan Zhu J. Phys. D: Appl. Phys. 2013 46(6), 65302.doi:10.1088/0022-3727/46/6/065302.

[20]Kejie Fang, Zongfu Yu, Victor Liu, and Shan hui Fan. Optics Letters. 2011 36(21), 4254.doi:10.1364/OL.36.004254.

[21]Xuan-Zhang Wang and D.R.Tilley. Phys. Rev. B. 1994 50(18), 13472.doi: 10.1103/PhysRevB.50.13472.

[22] Sheng Zhou, Hua Li, Shu-Fang Fu, and Xuan-Zhang Wang. Phys. Rev. B. 2009 80(20), 205409.doi: 10.1103/PhysRevB.80.205409.

[23]Zhijun Sun, Xiaoliu Zuo, Tengpeng Guan, and Wei Chen. Optics Express. 2014 22(4), 4714.doi:10.1364/OE.22.004714.

[24]L. Martín-Moreno, F. J. García-Vidal, H. J. Lezec, K. M. Pellerin, T. Thio, J. B. Pendry, and T. W. Ebbesen. Phys. Rev. Lett. 2001 86(6), 1114. doi:10.1103/ PhysRevLett.86.114.

[25]K. Abraha and D. R. Tilley. Surf. Sci. Rep. 1996 24(5-6), 129. doi:10.1016/ 0167-5729(96)00003-9

[26]N. S. Almeida and D. L. Mills. Phys. Rev. B. 1988 37(7), 3400. doi:10.1103/ PhysRevB.37.3400. 


\section{Figure Captions:}

Fig. 1. Schematic of dielectric/perforated metal/antiferromagnetic sandwich layered structure exhibiting one-way extraordinary optical transmission.

Fig. 2. (a) Positive transmittance $t$, and (b) reverse transmission $t^{\prime}$. The unidirectional parameters $\Gamma=\Pi-\Pi^{\prime}$ are shown in (c).

Fig. 3. Unipolarity of PM structure for various (a) hole sizes and (b) hole spacings.

Fig. 4. One-way parameter, which is affected by the dielectric layer structure or incident directions, is represented by the color legend: One-way effect for (a) two dielectric materials, $\mathrm{ZrO}_{2}$ and $\mathrm{ZrF}_{2}$ ( $\varepsilon=8.0$ and $\varepsilon=27.0$, respectively) and (b) for two incident directions $\left(\theta=20^{\circ}\right.$ and $\left.\theta=80^{\circ}\right)$.

Fig. 5. Unipolarity dependence on layer thickness for $(a-c)$ dielectric, $P M$, and AF layers, respectively.

Fig. 6. Rotation as a function of magnetic field $H_{0}$ for other fixed parameters. The black line indicates the same frequency 


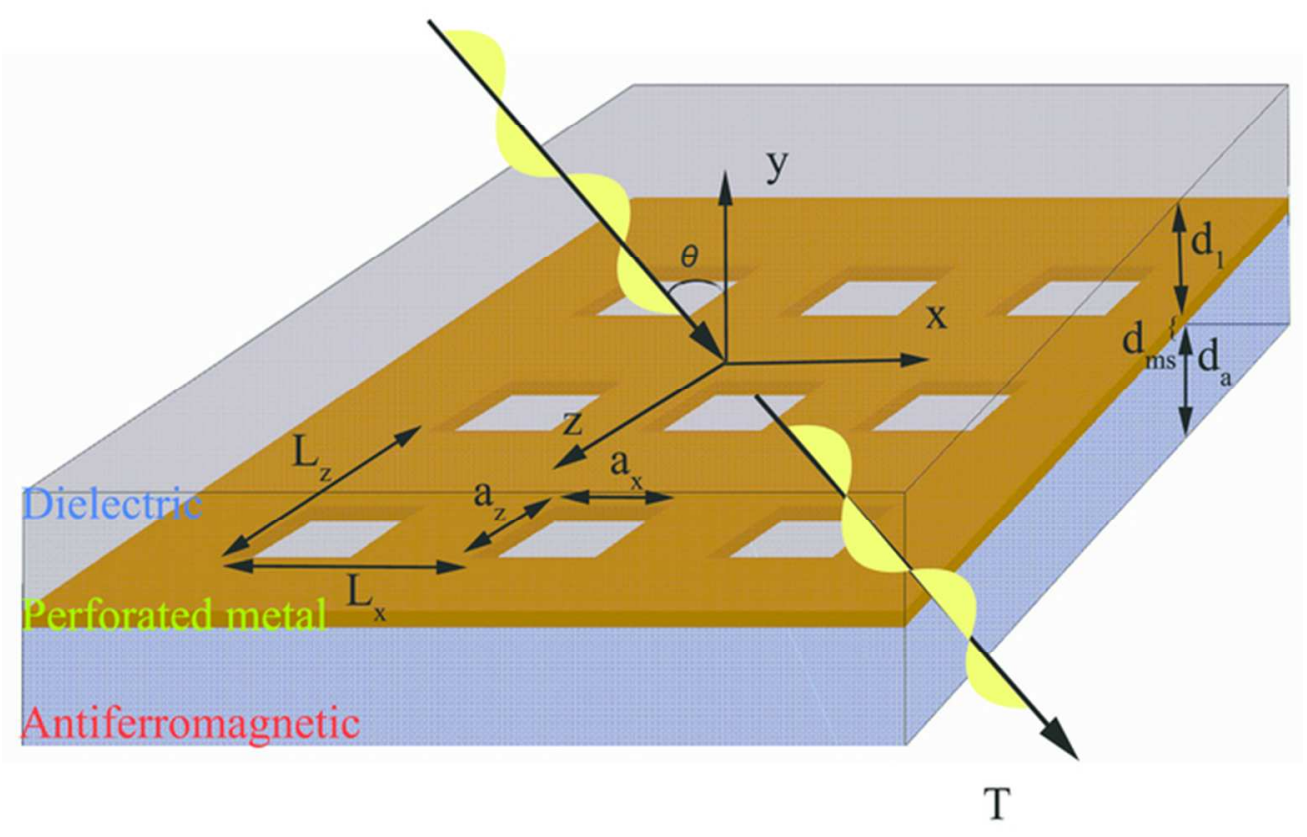

Fig. 1

$59 \times 42 \mathrm{~mm}(300 \times 300$ DPI $)$ 

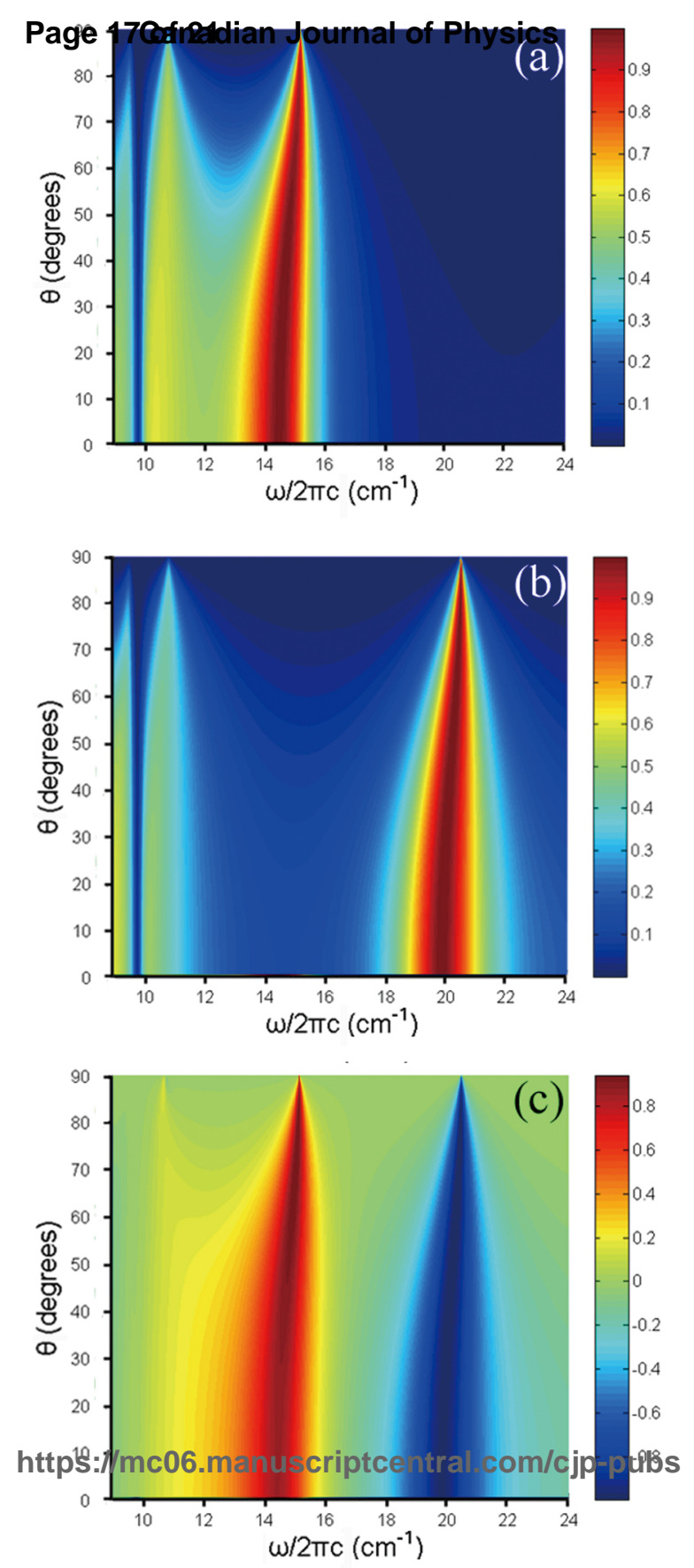


\section{Canadian Journal of PRagsec 58 of 21}
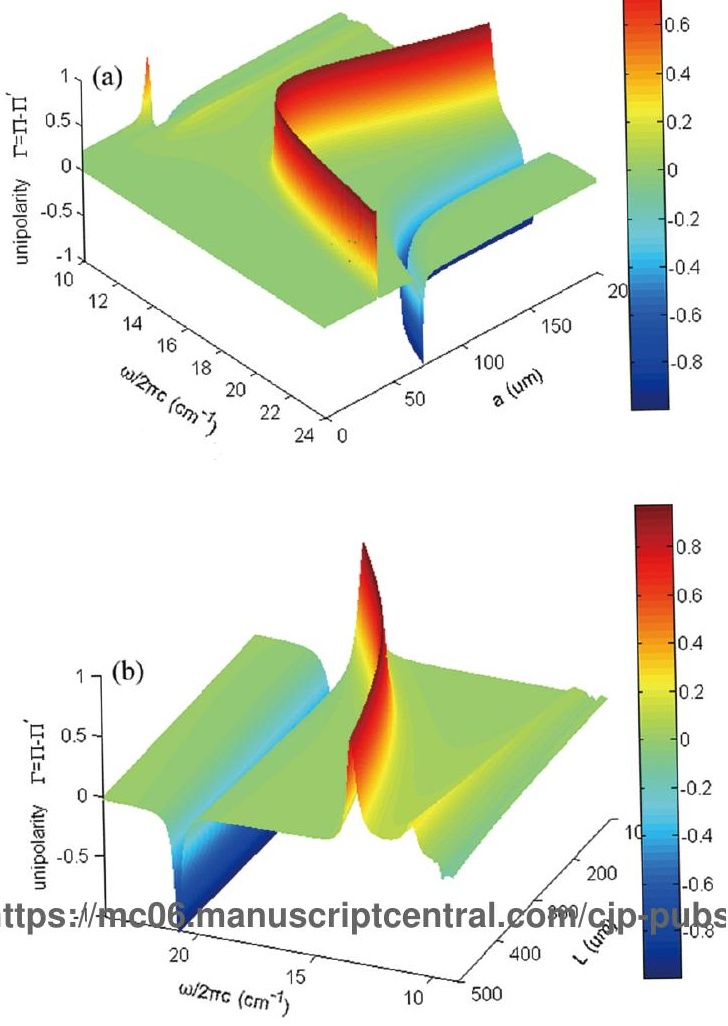


\section{Page 19Carpadian Journal of Physics}
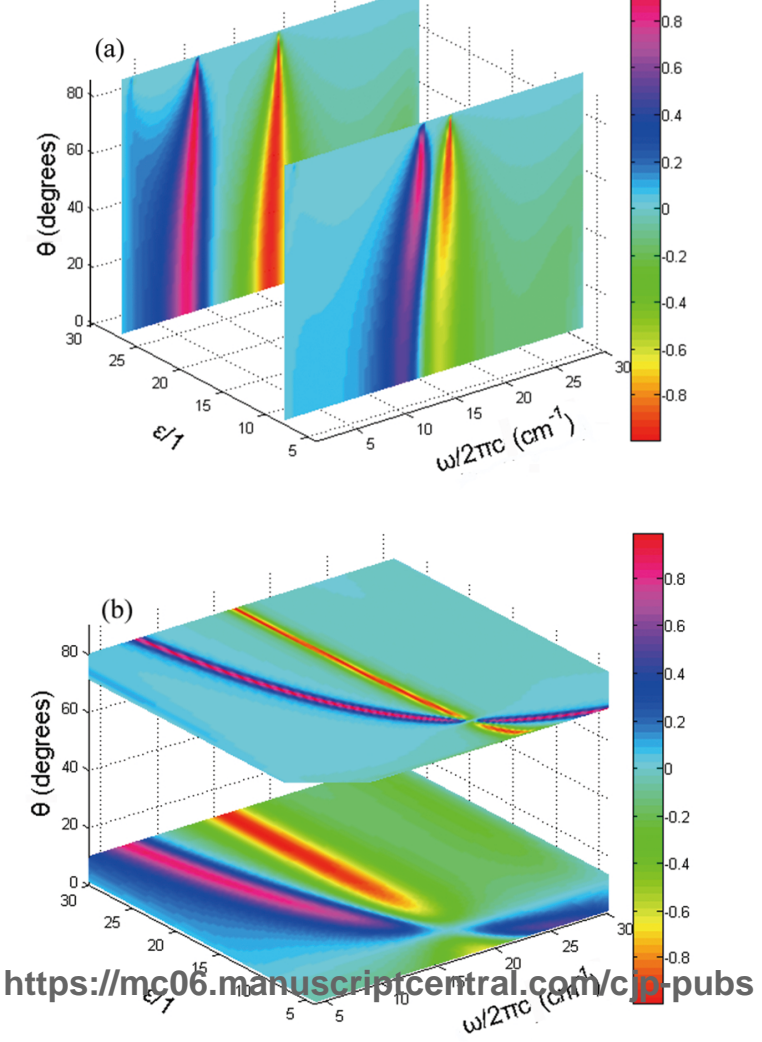

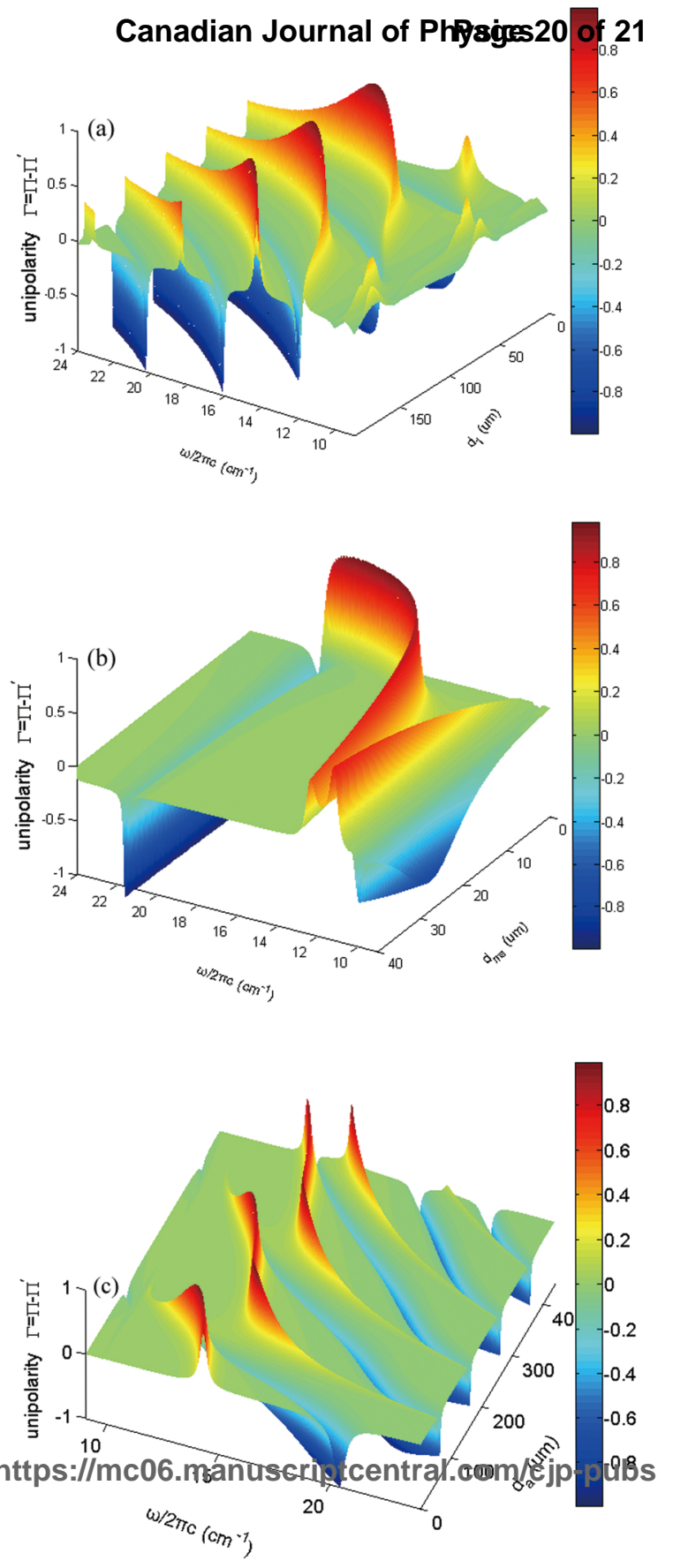


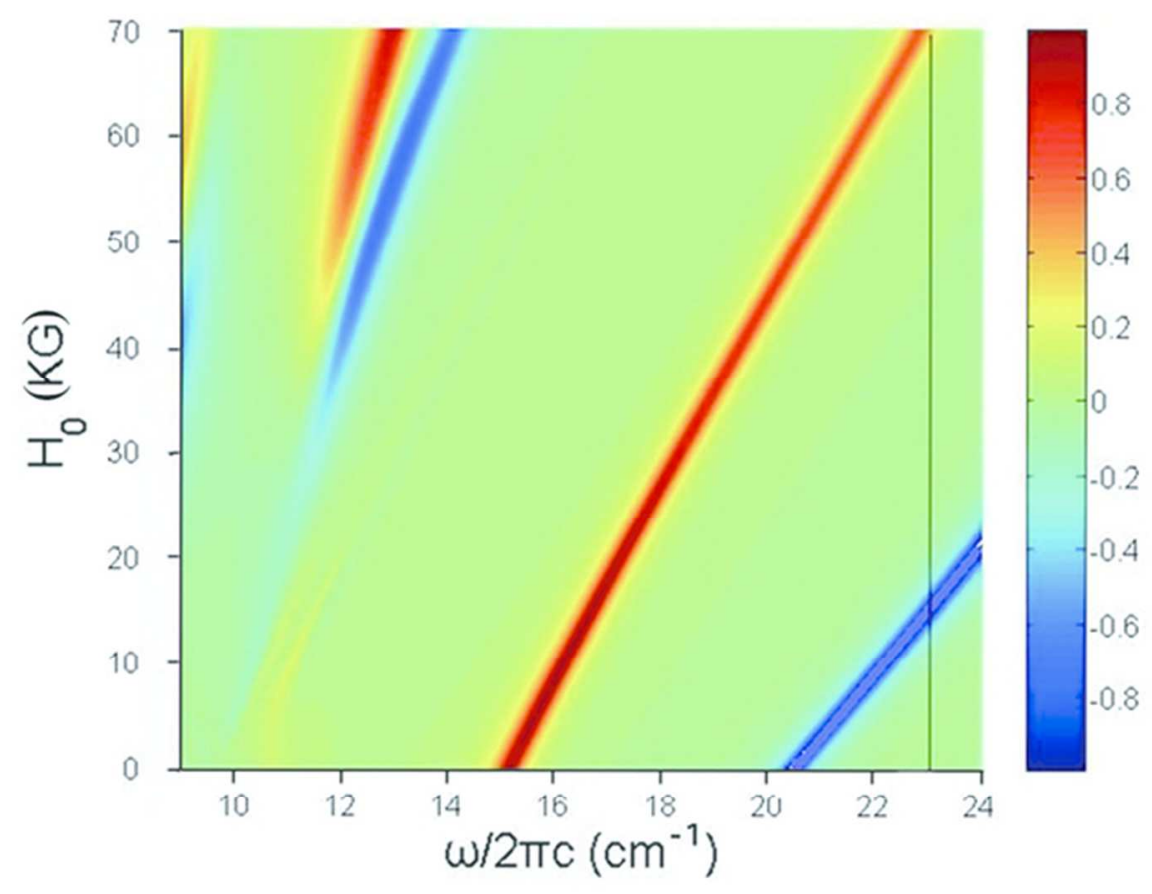

Fig. 6

$59 \times 44 \mathrm{~mm}(300 \times 300$ DPI $)$ 